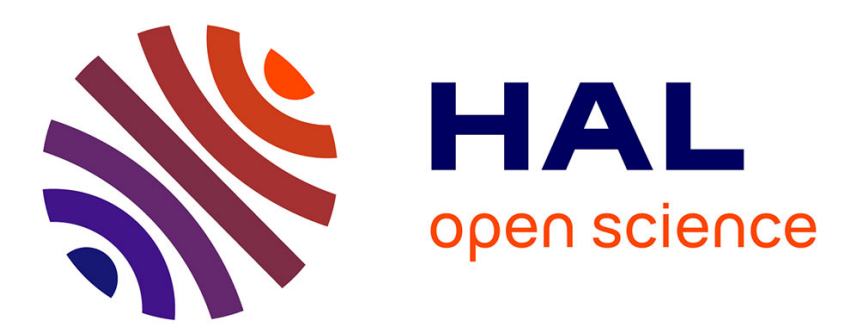

\title{
Optimal Control of a Vehicular Organic Rankine Cycle Via Dynamic Programming with Adaptive Discretization Grid
}

Johan Peralez, Paolino Tona, Antonio Sciarretta, Pascal Dufour, Madiha Nadri

\section{To cite this version:}

Johan Peralez, Paolino Tona, Antonio Sciarretta, Pascal Dufour, Madiha Nadri. Optimal Control of a Vehicular Organic Rankine Cycle Via Dynamic Programming with Adaptive Discretization Grid. IFAC Word Congress, Aug 2014, Cape Town, South Africa. pp.5671-5678, 10.3182/20140824-6-ZA1003.02185. hal-01097214

\section{HAL Id: hal-01097214 https://hal.science/hal-01097214}

Submitted on 28 Jan 2016

HAL is a multi-disciplinary open access archive for the deposit and dissemination of scientific research documents, whether they are published or not. The documents may come from teaching and research institutions in France or abroad, or from public or private research centers.
L'archive ouverte pluridisciplinaire $\mathbf{H A L}$, est destinée au dépôt et à la diffusion de documents scientifiques de niveau recherche, publiés ou non, émanant des établissements d'enseignement et de recherche français ou étrangers, des laboratoires publics ou privés. 
This document must be cited according to its final version

which is published in a conference proceeding as:

J. Peralez ${ }^{1,2}$, P. Tona ${ }^{2}$, A. Sciarretta ${ }^{3}$, P. Dufour ${ }^{1}$, M. Nadri ${ }^{1}$, 'Optimal Control of a vehicular organic rankine cycle via dynamic programming with adaptive discretization grid", IFAC World Congress, Cape Town, South Africa, pp. 5671-5678, August 24-29, 2014.

All open archive documents of Pascal Dufour are available at: http://hal.archives-ouvertes.fr/DUFOUR-PASCAL-C-3926-2008

The professional web page (Fr/En) of Pascal Dufour is: http://www.lagep.univ-lyon1.fr/signatures/dufour.pascal

The web page of this research group is: http://hal.archives-ouvertes.fr/SNLEP

1

Université de Lyon, Lyon, F-69003, France; Université Lyon 1;

CNRS UMR 5007 LAGEP (Laboratoire d'Automatique et de GEnie des Procédés),

$43 \mathrm{bd}$ du 11 novembre, 69100 Villeurbanne, France

Tel +33 (0) 472431845 - Fax +33 (0) 472431699

http://www-lagep.univ-lyon1.fr/ http://www.univ-lyon1.fr http://www.cnrs.fr

2

IFP Energies nouvelles - Control, Signal and System Department

Rond-point de l'échangeur de Solaize, BP 369360 Solaize, France

(e-mail: \{paolino.tona\}@ifpen.fr)

3

IFP Energies nouvelles - Control, Signal and System Department

$1 \& 4$, avenue de Bois-Préau 92852 Rueil-Malmaison Cedex, France

(e-mail: antonio.sciarretta@ifpen.fr) 


\title{
Optimal Control of a Vehicular Organic Rankine Cycle via Dynamic Programming with Adaptive Discretization Grid
}

\author{
J. Peralez ${ }^{*}$ P. Tona * A. Sciarretta ${ }^{* *}$ P. Dufour ${ }^{* * *}$ \\ M. Nadri ${ }^{* * *}$ \\ * IFP Energies nouvelles - Control, Signal and System Department \\ Rond-point de l'échangeur de Solaize, BP 369360 Solaize, France \\ (e-mail: \{johan.peralez, paolino.tona\}@ifpen.fr) \\ ** IFP Energies nouvelles - Control, Signal and System Department \\ 18 4, avenue de Bois-Préau 92852 Rueil-Malmaison Cedex, France \\ (e-mail: antonio.sciarretta@ifpen.fr) \\ *** Université de Lyon, F-69003, France - Université Lyon 1 - CNRS \\ UMR 5007 - Laboratory of Process Control and Chemical Engineering \\ (LAGEP) - 43 bd du 11 novembre, 69100 Villeurbanne, France \\ (e-mail: $\{$ dufour, nadri\}@lagep.univ-lyon1.fr)
}

\begin{abstract}
This paper addresses the problem of maximizing the power produced by an organic rankine cycle (ORC) waste heat recovery (WHR) system along a given vehicle mission. A simplified model of the system allows the formulation of an optimal control problem that can be solved via dynamic programming (DP). To increase the smoothness and the accuracy of the solution obtained using the state-of-the-art implementation of DP known as level-set DP, an improved version is developed, making use of adaptive grids for discretization. The adaptivegrid algorithm is first validated on a simple problem with an analytical solution, then applied to the more complex ORC case study. It is shown that the required accuracy can be achieved with a lower computational effort than the original algorithm. The analysis of the resulting optimal trajectory for the ORC control problem provides useful insight for both control design and system design.
\end{abstract}

Keywords: Dynamic programming, backward reachability, waste heat recovery, Rankine cycle, vehicle energy management.

\section{INTRODUCTION}

Over the past few decades, several problems of transient control of energy production on board vehicles in the presence of constraints have been addressed and solved via optimal control methods and in particular via dynamic programming (Bellman [1957]). A typical example is the energy management (EM) problem in hybrid electric vehicles (HEVs), which consists in minimizing a cost function (most often, fuel consumption) during vehicle operation while respecting driver's demand and actuator limitations, following a prescribed battery state-of-charge trajectory (Guzzella and Sciarretta [2007]; Serrao et al. [2011]). Although it is not directly implementable in real-time (as it needs perfect prediction of future driving cycle), dynamic programming proves very useful in this context, as an analysis tool to understand (optimal) HEV EM behaviour, as a benchmark to assess other EM strategies or as a method to determine the potential of a given architecture.

\footnotetext{
* The authors wish to thank ANR, the French National Research Agency, for partially funding this work within the framework of the project TRENERGY ( Train Energy Efficiency via Rankine-cycle Exhaust Gas Heat Recovery", \# ANR-12-VPTT-09), and ALSTOM Transport, for providing application data.
}

With new technologies continuously being introduced to further reduce vehicle fuel consumption, new control problems arise that must be efficiently solved to make the most of the innovation potential. This is for instance the case of engine waste heat recovery (WHR) systems based on the Rankine thermodynamic cycle. Applications of this technology exists, at different levels of maturity, for cars, trucks, trains and ships (see Sprouse and Depcik [2013] for a recent overview). For some applications, such as WHR via organic rankine cycle (ORC) from Diesel engines of long haul trucks, very interesting fuel consumption reductions have been reported (up to 6\%, according to Stanton [2013]). As shown in Endo et al. [2007], and more recently in Peralez et al. [2012], Peralez et al. [2013] and Xie and Yang [2013], control of Rankine-based WHR systems for vehicular applications is far from being trivial, especially when not addressed at initial stages of system design. On the other hand, an early assessment of the best dynamic performance achievable on a given architecture, and of the way to obtain it, can significantly facilitate control design, while providing valuable insight into the system.

This paper addresses the problem of maximizing the power produced by an ORC WHR system along a given vehicle mission. A simplified model of the system allows the for- 
mulation of an optimal control problem that can be solved via dynamic programming. However, despite the small size of the model, numerical issues linked to discretization slow down the search for an appropriate parameterization of the dynamic programming algorithm. To increase the smoothness and the accuracy of the solution obtained using the state-of-the-art implementation of DP known as Level-Set DP (Elbert et al. [2013]), an improved version is developed and validated on a simpler problem with an analytical solution, before application to the ORC case study.

We start by recalling the principle of dynamic programming, its basic formulation and the level-set algorithm (Section 2). In Section 3, we present our improved variant of the level-set algorithm obtained via an adaptive discretization grid. The new algorithm is first validated on a simple energy management example, where the power consumption of an electrical vehicle is to be minimized (Section 4). At the expense of an acceptable increase of computational load, the adaptive-grid DP algorithm indeed provides a smoother and more accurate solution.

In Section 5, we present the optimal control application consisting in maximizing the power produced by an organic Rankine cycle waste heat recovery system on board a diesel-electric train. First, we describe the system. Then we provide an experimentally validated simplified model, and use it to formulate the optimal control problem. We show that, for this application, the adaptive-grid DP algorithm achieves the same accuracy of the reference level-set DP algorithm with fewer model calls, while using a lower level of discretization.

The analysis of the resulting optimal control trajectory provides useful insight for both control design and system design.

\section{DYNAMIC PROGRAMMING AND LEVEL-SET METHOD}

The generic optimal control problem we want to solve for a dynamic system with $n$ state variables and $m$ control inputs can be written:

$$
\begin{aligned}
\min _{u(t)} J(u(t)) & \\
\text { s.t. } & \\
\dot{x}(t) & =f(x(t), u(t), t) \\
x(0) & =x_{0} \\
x(t) & =\mathcal{X}(t) \subseteq \mathbb{R}^{n} \\
u(t) & =\mathcal{U}(t) \subseteq \mathbb{R}^{m}
\end{aligned}
$$

where

$$
J(u(t))=g\left(x\left(t_{f}\right)\right)+\int_{0}^{t_{f}} h(x(t), u(t), t) d t,
$$

is the cost functional.

Since dynamic programming is a numerical algorithm, discretization of time, state space, and control space is required. Let the discrete-time model be

$$
x_{k+1}=f_{k}\left(x_{k}, u_{k}\right), k=0,1, \ldots, N-1
$$

where $x_{k} \in \mathcal{X}_{k}$ is the state vector and $u_{k} \in \mathcal{U}_{k}$ the control signal vector.

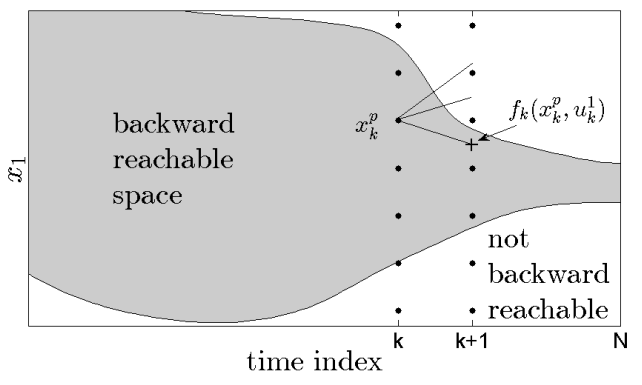

Fig. 1. Numerical issues near boundary surface

\subsection{Basic DP}

Based on principle of optimality introduced by Bellman [1957], DP proceeds backwards in time to evaluate the optimal cost-to-go function $\mathcal{J}_{k}\left(x^{i}\right)$ at every node $x^{i}$ of the discretized time-state space:

I. Final time computation

$$
\mathcal{J}_{N}\left(x^{i}\right)=\left\{\begin{array}{l}
g_{N}\left(x^{i}\right), \text { for } x^{i} \in \mathcal{X}_{N} \\
\infty \text { else. }
\end{array}\right.
$$

II. Intermediate computations for $k=N-1$ to 0

$$
\mathcal{J}_{k}\left(x^{i}\right)=\min _{u_{k} \in U_{k}}\left\{g_{k}\left(x^{i}, u_{k}\right)+\mathcal{J}_{k+1}\left(f_{k}\left(x^{i}, u_{k}\right)\right)\right\} .
$$

The optimal control is the argument that minimizes the right-hand side of equation (9) for each $x^{i}$ at time index $k$, which yields the optimal control policy $\pi=$ $\left\{\mu_{0}(x), \mu_{1}(x), \ldots, \mu_{N-1}(x)\right\}$. This map is used to find the optimal control signal during a forward simulation of model (7) starting from a given initial state $x_{0}$. Since the resulting state trajectory do not generally coincide with the nodes $x^{i}$, interpolation is used.

An major issue to consider is the definition of the costto-go function for infeasible states. Grid points that are not backward-reachable should have infinite cost as in (8). However, this causes numerical problems as illustrated in Fig. 1. When running step $k$ of backward iteration, the cost-to-go $\mathcal{J}_{k+1}(x)$ is known for all grid points $x^{i}$. In order to evaluate the cost-to-go $\mathcal{J}_{k}\left(x^{p}\right)$, all possible control candidates $u \in \mathcal{U}_{k}$ are applied. In Fig. 1, the point $f_{k}\left(x^{p}, u^{1}\right)$ is in the backward-reachable space. But since the value of $J_{k+1}\left(x^{i+1}\right)$ is infinite, interpolation will lead to consider that the cost-to-go of $x_{k}^{i+2}$ is infinite too.

A method to deal with this problem consists in using a large but finite value for infeasible states. The value of this penalty represents a critical parameter that is difficult to calibrate. Nevertheless, the penalty method results in a steep gradient of the cost-to-go function near the boundary and cannot completely solve the aforementioned problem.

\subsection{Level-set algorithm}

Another approach is based on the concept of backwardreachable space, as introduced in Back et al. [2004]; Sundström et al. [2010]. More particularly, the level-set DP algorithm, introduced by Elbert et al. [2013], evaluates the backward-reachable space in parallel with cost-to-go function evaluation. A level-set function $\mathcal{I}$ is introduced that associates a real value to the state $x$ :

$$
\mathcal{I}: X \subseteq \mathbb{R}^{n} \rightarrow \mathbb{R}
$$


where $\mathcal{I}(x) \leq 0$ if $x$ is in reachable space and $\mathcal{I}(x)>0$ else. The level-set function is evaluated for grid points $x^{i}$ at each time index $k$ yielding a grid evaluation $\mathcal{I}_{x}^{k}$. At the next backward iteration step $k+1$, interpolation is used to determine whether a point $x$ is backward reachable or not. Such a function can represent nonconvex regions, moreover, the number of state and control inputs is not limited. Assume that the final state constraint is given as a target set $T$, which is defined by a level-set function $h(x)$

$$
\begin{aligned}
h: X_{N} & \rightarrow \mathbb{R}, \text { where } X_{N} \subseteq \mathbb{R}^{n} \\
T & =\left\{x \in X_{N} \mid h(x) \leq 0\right\} .
\end{aligned}
$$

Then a DP algorithm is applied where the cost-to-go function to minimize is the level-set function $\mathcal{I}$ :

\section{Initialization}

$$
\mathcal{I}_{N}\left(x^{i}\right)=h\left(x^{i}\right) .
$$

II. Backward iteration for $k=N-1$ to 0

$$
\mathcal{I}_{k}\left(x^{i}\right)=\min _{u_{k} \in U_{k}}\left\{\mathcal{I}_{k+1}\left(f_{k}\left(x^{i}, u_{k}\right)\right)\right\} \text {. }
$$

\section{ADAPTIVE-GRID DP ALGORITHM}

\subsection{Backward algorithm}

The level-Set DP algorithm introduced in Elbert et al. [2013] can be modified to improve the accuracy of solution.

I. Initialize $\mathrm{k}=\mathrm{N}$ and the level-set and cost-to-go functions as:

$$
\begin{array}{r}
I_{N}\left(x^{i}\right)=h\left(x^{i}\right) \\
J_{N}\left(x^{i}\right)=g_{N}\left(x^{i}\right) .
\end{array}
$$

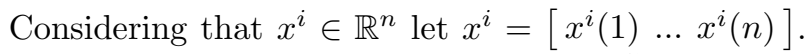
A second discretization of state space $\tilde{X}_{N}$ is introduced:

$$
\tilde{X}_{N}=\left\{\tilde{x}_{N}^{1}, \tilde{x}_{N}^{2}, \ldots, \tilde{x}_{N}^{q}\right\}
$$

where each $j^{\text {th }}$ component of vector $\tilde{x}_{N}^{i}$ is bounded below by $\underline{x}_{N}(j)$ and above by $\bar{x}_{N}(j)$ as follows:

$$
\begin{aligned}
& \underline{x}_{N}(j)=\min _{i \in\{1, \ldots, q\}}\left\{x_{N}^{i}(j) \mid 0<\mathcal{I}_{N}\left(x_{N}^{i}\right)\right\} \\
& \bar{x}_{N}(j)=\max _{i \in\{1, \ldots, q\}}\left\{x_{N}^{i}(j) \mid 0<\mathcal{I}_{N}\left(x_{N}^{i}\right)\right\}
\end{aligned}
$$

As illustrated in Fig. 2 this second discretization may improve the accuracy of level-set and cost-to-go estimations:

$$
\begin{array}{r}
\tilde{I}_{N}\left(\tilde{x}^{i}\right)=h\left(\tilde{x}^{i}\right) \\
\tilde{J}_{N}\left(\tilde{x}^{i}\right)=g_{N}\left(\tilde{x}^{i}\right) .
\end{array}
$$

II. Reduce $k$ by 1 and update the level-set function by

$$
I_{k}\left(x^{i}\right)=\min _{u_{k} \in U_{k}}\left\{\tilde{I}_{k+1}\left(f_{k}\left(x^{i}, u_{k}\right)\right)\right\} .
$$

A second discretization $\tilde{X}_{k}$ is computed in the same way as for the first step. Then the level-set function is updated by

$$
\tilde{I}_{k}\left(\tilde{x}^{i}\right)=\min _{u_{k} \in U_{k}}\left\{\tilde{I}_{k+1}\left(f_{k}\left(\tilde{x}^{i}, u_{k}\right)\right)\right\}
$$

III. For each grid point $\tilde{x}^{i}$, find the set of control signals for which the system trajectory ends up inside the backward-reachable space at the next time step

$$
U_{k}^{F}\left(x^{i}\right)=\left\{u_{k} \in U_{k} \mid \tilde{I}_{k+1}\left(f_{k}\left(\tilde{x}^{i}, u_{k}\right)\right) \leq 0\right\},
$$

and the one control candidate that minimizes the level-set function

$$
\stackrel{*}{u}_{k}\left(\tilde{x}^{i}\right)=\arg \min _{u_{k} \in U_{k}}\left\{\tilde{I}_{k+1}\left(f_{k}\left(\tilde{x}^{i}, u_{k}\right)\right)\right\} .
$$

IV. Update the optimal cost-to-go by the following rule: if at least one valid control candidate is found, i.e., $U_{k}^{F}\left(x^{i}\right) \neq \emptyset$, then calculate the cost-to-go based upon the optimal candidate

$$
\tilde{J}_{k}\left(\tilde{x}^{i}\right)=\min _{u_{k} \in U_{k}^{F}\left(x^{i}\right)}\left\{g_{k}\left(\tilde{x}^{i}, u_{k}\right)+\tilde{J}_{k+1}\left(f_{k}\left(\tilde{x}^{i}, u_{k}\right)\right)\right\} .
$$

If, however, the grid point is not backward-reachable, then calculate the cost-to-go based on the control input $\stackrel{*}{u_{k}}\left(\tilde{x}^{i}\right)$

$$
\tilde{J}_{k}\left(\tilde{x}^{i}\right)=g_{k}\left(\tilde{x}^{i}, \stackrel{*}{u}_{k}\right)+\tilde{J}_{k+1}\left(f_{k}\left(\tilde{x}^{i}, \stackrel{*}{u}_{k}\right)\right)
$$
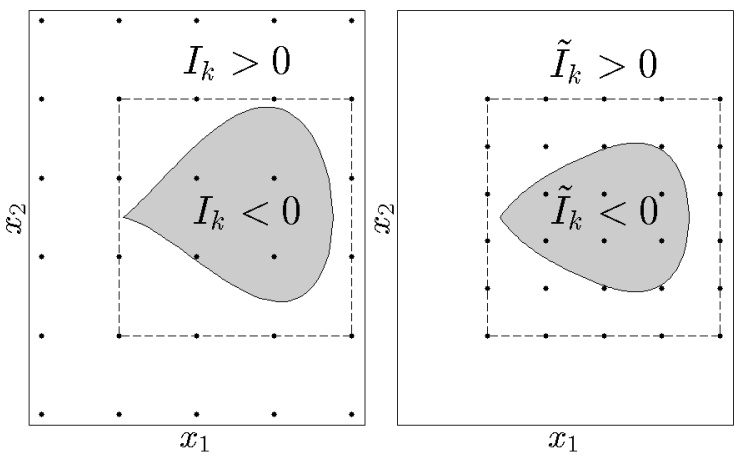

Fig. 2. A first estimation $I_{k}$ of level-set function is computed on the whole state space (left plot). A second discretization (right plot) allows to improve the accuracy of both level-set and cost-to-go functions.

\subsection{Forward algorithm}

To increase smoothness, the forward algorithm introduced in Elbert et al. [2013] is used with a new discretization of the control space:

$$
\tilde{U}_{k}=\{\underbrace{u_{k}^{1}, \ldots, u_{k}^{r}}_{U_{k}}, \ldots, u_{k}^{\tilde{r}}\}
$$

I. Initialize $k=0$ and $x_{0}$ at initial condition.

II. Increase $k$ by 1 and find the feasible control candidates

$$
\tilde{U}_{k}^{F}\left(x_{k}\right)=\left\{u_{k} \in \tilde{U}_{k} \mid \tilde{I}_{k+1}\left(f_{k}\left(x_{k}, u_{k}\right)\right) \leq 0\right\},
$$

III. Find the optimal control input

$$
u_{k}^{0}\left(x_{k}\right)=\arg \min _{u_{k} \in U_{k}}\left\{g_{k}\left(x_{k}, u_{k}\right)+\tilde{J}_{k+1}\left(f_{k}\left(x_{k}, u_{k}\right)\right)\right\}
$$

IV. Simulate the system using the optimal control input

$$
x_{k+1}=f_{k}\left(x_{k}, u_{k}^{o}\right)
$$

and repeat steps II. - IV. until $k=N$.

Note that the computational effort involved in the finer discretization $\tilde{U}_{k}$ is limited. Since steps II. - IV. of the algorithm are applied to one value of $x_{k}$, the number of additional calls to the model is $N \cdot(\tilde{r}-r)$. 


\section{A SIMPLE ENERGY MANAGEMENT EXAMPLE}

To illustrate the benefits of the new algorithm, a first optimal control problem with an analytic solution is studied. It concerns energy management for an electric vehicle.

\subsection{System description}

Neglecting the aerodynamic friction and considering the road slope null, the simplified continuous-time dynamics of the vehicle in the longitudinal direction $x$ can be written as in Petit and Sciarretta [2011]:

$$
\dot{x}=v, \dot{v}=h_{1} u-h_{0},
$$

where the control variable $u$ is a percent torque demand, $h_{0}$ and $h_{1}$ are constant parameters depending on the rolling resistance coefficient, the motor maximum torque, vehicle mass, transmission ratio and wheel radius.

The on-board electric power consumption can be written as

$$
P_{m}=b_{1} u v+b_{2} u^{2}
$$

\subsection{Optimal control problem}

We wish to find a control strategy that minimizes the power consumption under the constraints that the vehicle must reach a destination point at a distance $D$ in a given time $t_{f}$ starting from a given point, at rest. In order to formulate this problem for DP solving, the continuous-time model is discretized using an Euler forward approximation with a time step $T_{s}$. The optimal control problem can be formulated as follows:

$$
\begin{array}{r}
\min _{u_{k} \in[-2,2]} \sum_{k=0}^{N-1}\left(b_{1} u_{k} v_{k}+b_{2} u_{k}^{2}\right) T_{s} \\
v_{k+1}=v_{k}+\left(h_{1} u_{k}-h_{0}\right) T_{s} \\
x_{k+1}=x_{k}+v_{k} T_{s} \\
x_{0}=0, v_{0}=0 \\
0 \leq x_{k} \leq \bar{x}, 0 \leq v_{k} \leq \bar{v} \\
D \leq x_{N} \leq \bar{x}, 0 \leq v_{N} \leq \epsilon_{v},
\end{array}
$$

where $N=\frac{t_{f}}{T_{s}}$. The final constraints are formulated in equations (39). In the following, a particular solution is sought for a final distance $D=200$ and a final time $t_{f}=60$. The time discretization is chosen to be $T_{s}=0.2 \mathrm{~s}$ (then $N=300$ ), with $\bar{x}=205, \bar{v}=8, \epsilon_{v}=0.3$.

\subsection{DP solution}

The problem stated above is solved using the basic, the original level-set and the level-set with two-step discretization DP algorithms.

State variable spaces are first discretized respectively with $N_{v}=81$ and $N_{x}=106$, while the control space is discretized with $N_{u}=81$. Fig. 3 shows the resulting system trajectory and the corresponding control inputs for each of the three algorithms. They are compared to the analytic solution (a parabola) presented by Petit and Sciarretta [2011]. The results obtained using the new algorithm are closer to the analytic solution, even with a

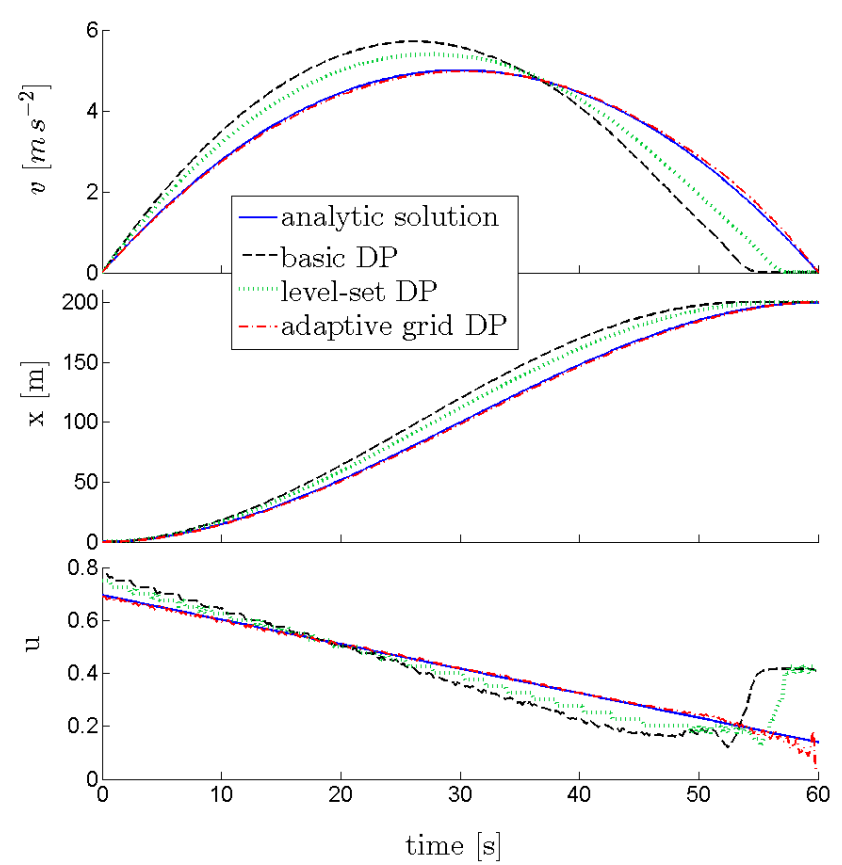

Fig. 3. Vehicle trajectory and control inputs when solving the problem with $N_{v}=81, N_{x}=106$ and $N_{u}=81$

relatively coarse discretization. In term of cost, the relative mean errors of the three algorithms are found to be of $1.4 \%, 0.78 \%$ and $0.19 \%$ respectively.

The evolution of backward-reachable space boundaries, where level-set function vanishes, helps to analyze those results. Fig. 4 shows that in the first backward steps, the optimal trajectory $\left(x^{o}, v^{o}\right)$ is close to $\tilde{I}_{k}$ estimations. Here, for accurate estimation of boundaries it is important not to consider reachable points close to the optimal solution as infeasible. After those first backward steps, the optimal trajectory moves away from the boundaries. However, the reachable state space remain restricted. Thus, the second discretization step of the proposed algorithm can improve the accuracy of cost-to-go function.

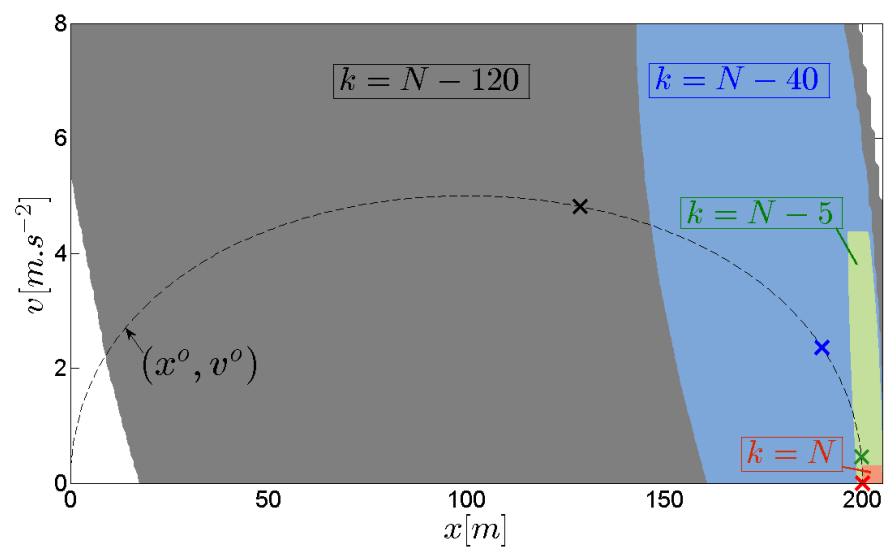

Fig. 4. Optimal trajectory $\left(x^{o}, v^{o}\right)$ and evolution of backward-reachable space (evaluated by adaptive-grid DP).

Two-step discretization involves a larger computational effort for a given set of parameters $\left(N_{v}, N_{x}, N_{u}\right)$. This computation cost can be expressed in terms of number 


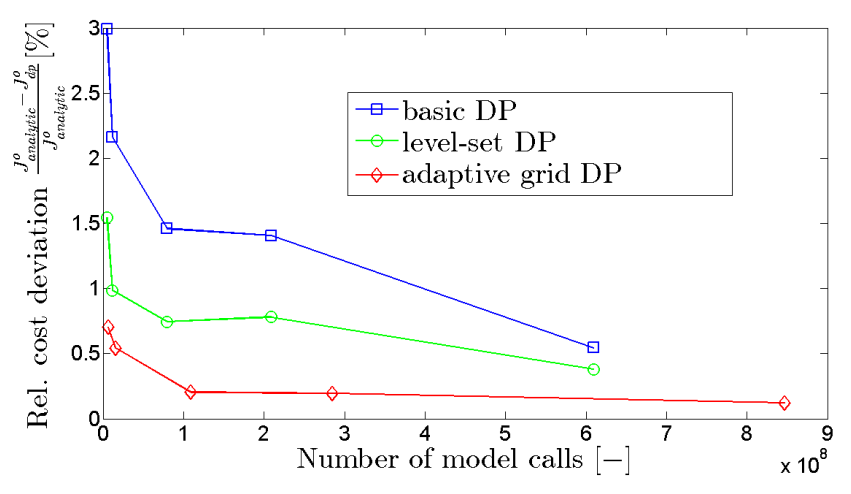

Fig. 5. Algorithm accuracies vs number of model calls for the simple energy management example.

of model calls for evaluation. For example, for the level of discretization used above, basic and original level-set DP both require about $2.085 \cdot 10^{8}$ model calls whereas the new algorithm requires about $2.85 \cdot 10^{8}$. For a fair comparison, algorithm accuracies are compared for different levels of discretization. Results are shown in Fig. 5. The adaptivegrid DP algorithm attains acceptable accuracies for a much lower number of calls.

\section{OPTIMAL CONTROL OF AN ORC FOR WHR ON BOARD TRAINS}

\subsection{System description}

The system under consideration is represented in Fig. 6. It is a prototype ORC system for WHR from a diesel engine generating set ("Power Pack") manufactured by Alstom Transport for installation in diesel-electric railcars. A pump pressurizes an organic fluid (R365mfc) in liquid state and circulates it through a closed circuit. Via a bypass valve, a fraction $V_{o, e}$ of engine exhaust gas is fed to a heat exchanger (evaporator) which transfers heat to the working fluid. The vaporized fluid then enters a turbine, which converts some of the kinetic energy into work, driving a generator connected to the Power Pack electrical network. Lastly, the fluid is recondensed before returning to the pump at low pressure. Cooling is provided by a fan with controllable air flow $\stackrel{*}{m}_{A}$.

The objective here is to find the controls $u=\left\{\stackrel{*}{m}_{A}, V_{o, e}\right\}$ that maximize the net power $P_{\text {net }}$ produced by the WHR system, function of $P_{\text {exp }}$, the power recovered by the turbine, and of $P_{\text {pump }}$ and $P_{A}$, the powers consumed by the pump and the cooling system :

$$
P_{n e t}=P_{e x p}-P_{\text {pump }}-P_{A},
$$

The thermodynamic cycle of the working fluid is represented in Fig. 7. In the following, subscripts 1 to 4 will refer to the working fluid at components outlet, namely the evaporator, the expander, the condenser and the pump.

Recoverable mechanical power from the turbine is usually expressed (Bao and Zhao [2013]) in terms of a constant efficiency $\eta_{\text {exp }}$, of the working fluid mass-flow $\stackrel{*}{m}$ and of the enthalpy gradient $h_{2}-h_{1}$ :

$$
P_{\text {exp }}=\stackrel{*}{m}\left(h_{2}-h_{1}\right) \eta_{\exp } .
$$

Similarly, the efficiency $\eta_{\text {pump }}$ can be considered constant and the power consumed by the pump can be written in terms of the pressure gradient and the fluid density:

$$
P_{\text {pump }}=\stackrel{*}{m} \frac{p_{1}-p_{3}}{\rho_{3} \eta_{\text {pump }}} .
$$

The power consumed by the fan can be considered proportional to the delivered air flow (Manente et al. [2013]):

$$
P_{A}=\stackrel{*}{m}_{A} k_{A} .
$$

To solve this problem, we need a model to predict the evolution of the variables in the expressions of powers (41)(43), that is fluid mass-flow, pressures and enthalpies.

Model dimensions should be small so as not to be confronted with the well-known "curse of dimensionality" (Powell [2007]). The so-called moving boundaries (MB) method allows a realistic dynamic representation of heat exchangers with a limited number of states. Cheng et al. [2004] proposes a model that considers an average wall temperature, while Peralez et al. [2013] shows experimentally that, for an ORC, the slowest dynamics are those of the wall temperatures. Thus, it is possible to neglect the fluid dynamics, thereby reducing the number of states.

In the next paragraph, we propose a new heat exchanger model with a single state variable obtained by applying the first law of thermodynamics. This model is then validated against experimental data, showing that it is able to capture the slowest dynamics of the ORC.

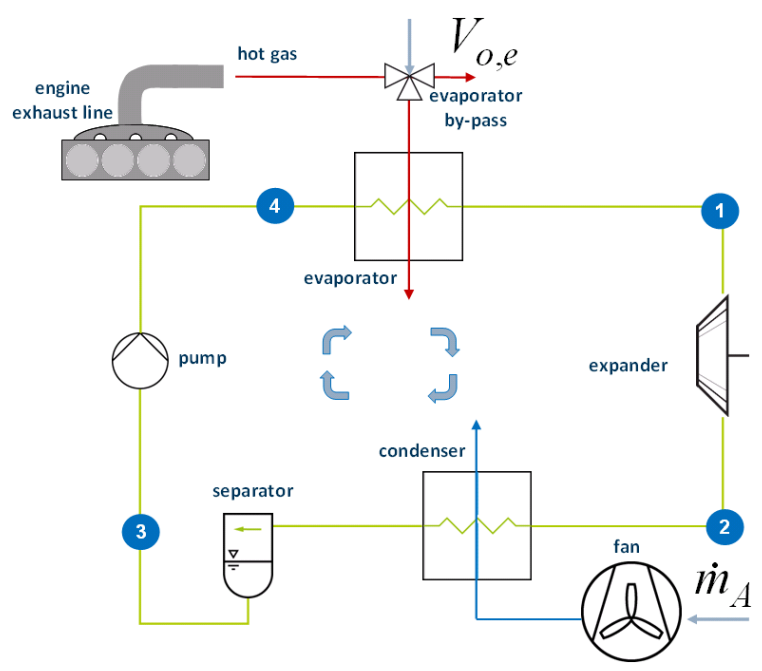

Fig. 6. ORC system under investigation

Two-state ORC model. Considering the thermodynamic cycle in Fig. 7, fluid superheating at evaporator outlet (point 1) is assumed to be perfectly regulated at a constant value by the pump mass-flow. The fluid at pump inlet (point 3) is assumed to be in the saturated liquid state. The slow dynamics of the system, i.e. the thermal dynamics of exchangers walls, are taken into account while the fluid is considered to be in equilibrium.

Let $\bar{T}_{w, e}$ and $\bar{T}_{w, c}$ the average wall temperatures respectively of the evaporator and the condenser. Let $\bar{T}_{f, e}$ and $\bar{T}_{f, c}$ the average temperatures of the working fluid circulating respectively in the evaporator and the condenser. The wall energy balance can be written as: 


$$
\begin{aligned}
M_{w, e} \dot{\bar{T}}_{w, e}= & S_{f, e} \bar{\alpha}_{f, e}\left(\bar{T}_{f, e}-\bar{T}_{w, e}\right) \\
& +V_{o, e} \stackrel{*}{m e x h}_{e p} c p_{e x h}\left(T_{e x h}-\bar{T}_{w, e}\right) \\
M_{w, c} \dot{\bar{T}}_{w, c}= & S_{f, c} \bar{\alpha}_{f, c}\left(\bar{T}_{f, c}-\bar{T}_{w, c}\right) \\
& +\eta_{A} \stackrel{*}{m}_{A} c p_{A}\left(T_{A}-\bar{T}_{w, c}\right),
\end{aligned}
$$

where $\eta_{A}=1-\exp \left(-\frac{\alpha_{A} S_{A}}{\stackrel{*}{m}_{A} c_{A}}\right)$ reflects the decrease in heat exchange efficiency for large air mass flows (McKinley and Alleyne [2008]).

In the following, $\bar{T}_{f, e}$ and $\bar{T}_{f, c}$ will be assumed equal to the evaporation temperatures respectively of the high-pressure and of the low-pressure sides of the ORC. $\bar{T}_{f, e}\left(\operatorname{resp} . \bar{T}_{f, c}\right)$ is then an unimodal function of $p_{1}$ (resp. of $p_{3}$ ). Likewise, $h_{1}, \rho_{1}$ depend only on $p_{1}$, whereas $h_{3}$ depends on $p_{3}$.

Fluid energy balances in the exchangers yield:

$$
\left\{\begin{array}{c}
0=h_{1}-S_{f, e} \bar{\alpha}_{f, e} \frac{\bar{T}_{w, e}-\bar{T}_{f, e}}{m_{m}}-h_{3} \\
0=h_{3}-S_{f, c} \bar{\alpha}_{f, c} \frac{\bar{T}_{w, c}-\bar{T}_{f, c}}{m}-h_{2},
\end{array}\right.
$$

where the mass flow $\stackrel{*}{m}$ is considered homogeneous throughout the circuit. The mass flow through the turbine is given by

$$
\stackrel{*}{m}=k \sqrt{2 \rho_{1} p_{1}},
$$

where $k$ is a constant (function of the equivalent section of the turbine nozzles).

Assuming a constant isentropic efficiency $\eta_{i s}$ of the turbine

$$
h_{2}=h_{1}-\eta_{i s}\left(h_{1}-h_{i s, 2}\right) \text {, }
$$

where $h_{i s, 2}$ corresponds to the enthalpy whose entropy is equal to that of point 1 and pressure is equal to that of point 3 . $h_{i s, 2}$ then depends on $p_{1}$ and $p_{3}$.

Using the previous expressions for $\stackrel{*}{m}$ and $h_{2}$, system (45) can be written in the following implicit form:

$$
0=\Phi\left(\bar{T}_{w, e}, \bar{T}_{w, c}, p_{1}, p_{3}\right) .
$$

Knowing $\bar{T}_{w, e}$ and $\bar{T}_{w, c},(48)$ is solved numerically by iterating over $p_{1}$ and $p_{3}$ (as illustrated in Fig. 8). $\bar{T}_{f, e}$ and $\bar{T}_{f, c}$ are then deduced from $p_{1}$ and $p_{3}$ to express the wall thermal dynamics (44).

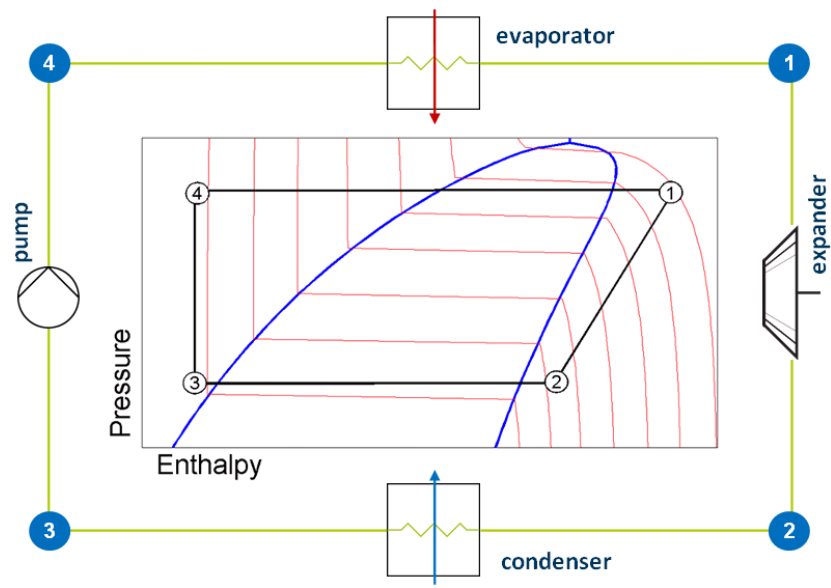

Fig. 7. Rankine cycle for "dry" fluid
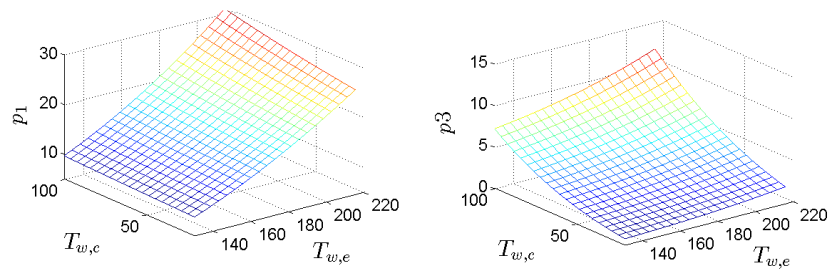

Fig. 8. $p_{1}$ and $p_{3}$ maps as function of $\bar{T}_{w, e}$ and $\bar{T}_{w, c}$

Experimental validation of the simplified model. In order to validate the heat-exchanger one-state model, the evaporator model response to transient external conditions is compared to experimental data. Transient conditions on exhaust gas entering in evaporator are shown in Fig. 9. Key measurement variables of working fluid at evaporator outlet, namely the mass flow $\stackrel{*}{m}$, the pressure $p_{1}$ and the temperature $T_{1}$ are plotted in Fig. 10. Mean relative deviations are found to be about $3.3 \%$ for working fluid mass flow, $2 \%$ for pressure and less than $1 \%$ for temperature.

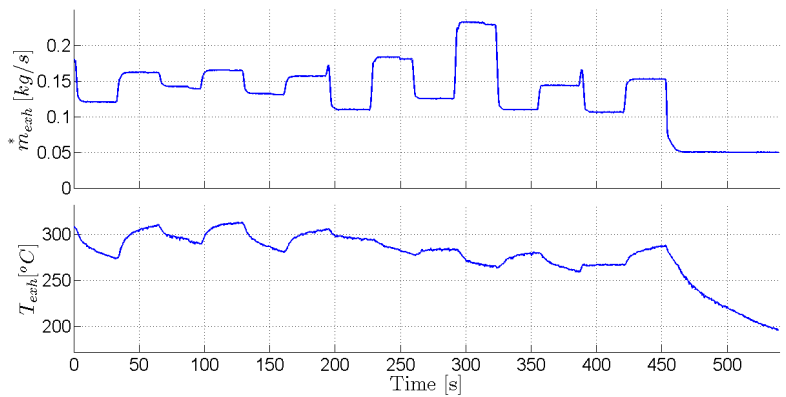

Fig. 9. External conditions for experimental validation of one-state evaporator model.

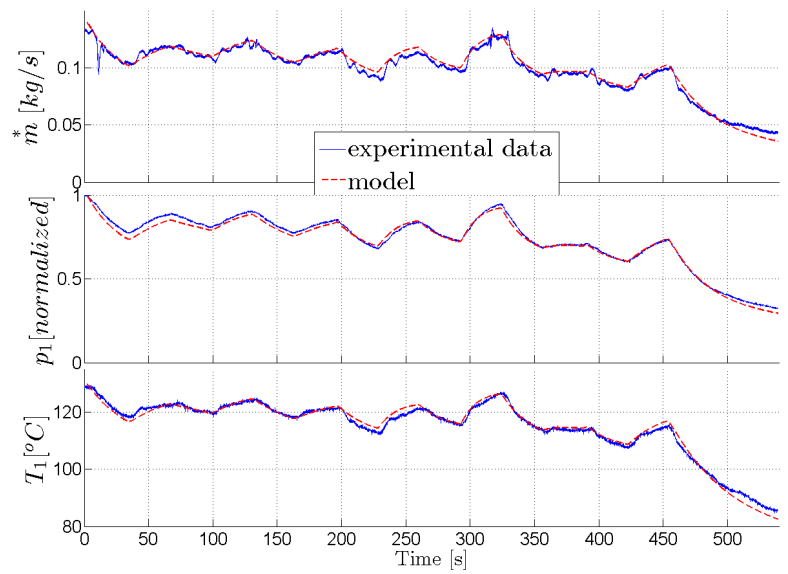

Fig. 10. One-state evaporator model: experimental validation

\subsection{Optimal control problem}

We wish to find a control strategy that maximizes the power production $P_{n e t}$, defined by equations (40)- (43), along a given mission of duration $t_{f}$. The mission profile considered herein, a portion of a typical intercity train trip, results in a set of external conditions for the ORC system, namely for the temperature $T_{\text {exh }}(t)$ and mass-flow $\stackrel{*}{m}$ exh $(t)$ 
Table 1. Comparison of computational efforts required for the ORC problem.

\begin{tabular}{ccc} 
& Level-set DP & Adaptive-grid DP \\
\hline Level of discretization & $N=24$ & $N=14$ \\
Mean net power produced & $5.485 \mathrm{~kW}$ & $5.497 \mathrm{~kW}$ \\
Number of model calls & $8.309 \cdot 10^{7}$ & $1.24 \cdot 10^{7}$ \\
\hline
\end{tabular}

of engine exhaust gas. A variation of ambient temperature $T_{A}(t)$ is also considered as shown in Fig. 11.

The cost functional to be maximized depends on time, state variables $x=\left\{\bar{T}_{w, e}, \bar{T}_{w, c}\right\}$ and control inputs $u=$ $\left\{V_{o, e}, \stackrel{*}{m}_{A}\right\}$ :

$$
J=\int_{0}^{t_{f}} P_{e x p}(x)+P_{\text {pump }}(x)+P_{A}(u, t), d t,
$$

where the dynamic of $x=\left\{\bar{T}_{w, e}, \bar{T}_{w, c}\right\}$ is defined by the two-state ORC model equations (44)-(48). This maximization problem must be solved under a security constraint on pressure $p_{1}$. A final constraint on wall temperature $x_{1}$ is also considered to let the system stop at the end of the mission:

$$
\begin{aligned}
p_{1}(x, t) & <25 \text { bar, } \forall t \in\left[0, t_{f}\right] \\
x_{1}\left(t_{f}\right) & <130{ }^{\circ} \mathrm{C}
\end{aligned}
$$

Bounds on control inputs are defined by technical considerations. The fraction of exhaust gas is bounded by $[0,1]$ whereas air mass flow provided by fan is comprised between 0 and $4 \mathrm{~kg} \mathrm{~s}^{-1}$ :

$$
u(t) \in[0,1] \times[0,4], \forall t \in\left[0, t_{f}\right]
$$

\subsection{DP solution}

The optimal control problem is solved using the original level-set DP and the new adaptive-grid DP algorithms. Different levels of state space and input variables discretization are tested with numbers of grid points set to $N=N_{x 1}=N_{x 2}=N_{u 1}=N_{u 2}$. The computational efforts - in terms of number of model evaluations - required to achieve a similar level of accuracy are compared in Table 1.

The comparison shows that, like in the simple thermal management example, a coarse discretization suffices to obtain good results using the adaptive-grid algorithm.

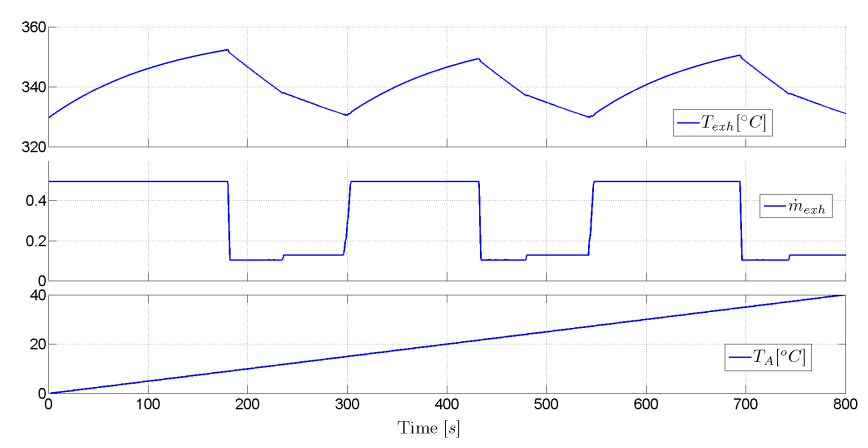

Fig. 11. Input disturbances for ORC problem.

\subsection{Results analysis}

Fig. 12 shows the system trajectory obtained with the discretization corresponding to $N=21$. Wall temperatures of heat exchangers periodically rise in response to the increase of the exhaust gas mass flow. This yields a pressure increase (top plot) activating the safety constraint (50). A the end of the mission, the final constraint (51) leads to the complete closing of the evaporator by-pass, i.e $u_{1}=0$.

The influence of ambient temperature on the overall efficiency of the ORC system is also highlighted. In fact, the power recovered by the turbine is substantially reduced for high ambient temperatures, whereas the powers consumed by the pump and the cooling fan are scarcely affected.

These results can be very useful to design a control strategy. We can infer that tracking control of condenser pressure can be obtained using the air mass flow $\stackrel{*}{m}_{A}$ to regulate condenser cooling; which would in turn ensure the pressure ratio required to run the turbine efficiently. On the other hand, the evaporator by-pass can be used to enforce safety limits in terms of pressure and temperature.

Solving the optimal control problem introduced above should also be useful at system design stage, especially considering that transient behavior is accounted for in system performance evaluation. The problem could be formulated for variants of the system under consideration, obtained with different sets of model parameters. For instance, the influence of working fluid choice or of exchangers sizing - taken into account in the two-state model described in Section 5.1 - could be studied. Thanks to the good accuracy achievable by the adaptive-grid DP algorithm at low levels of discretization, it seems feasible to study the impact of these (numerous) parameters within reasonable simulation times.

\section{CONCLUSION}

This paper proposes two main contributions.

- An original optimal control problem is formulated in order to find the maximum power produced by an organic Rankine cycle waste heat recovery system on board a diesel-electric train, along a given mission profile. An ad-hoc simplified model is developed, and experimentally validated, to make solving by dynamic programming computationally treatable. The optimal control trajectories obtained provide valuable insight into system behavior and prove useful for control design.

- An improved version of the state-of-the-art level-set DP algorithm is developed based on an adaptive grid, and validated on a simpler optimal control problem with an analytical solution, in order to achieve the required accuracy with a lower level of discretization and shorter computational time than the original algorithm.

Since the proposed improvement does not rely on a specific structure, application of the new algorithm should also be beneficial to other optimal control problems. Yet, we have not proven (nor tried to prove) that our "adaptivegrid" version consistently outperforms the original level- 


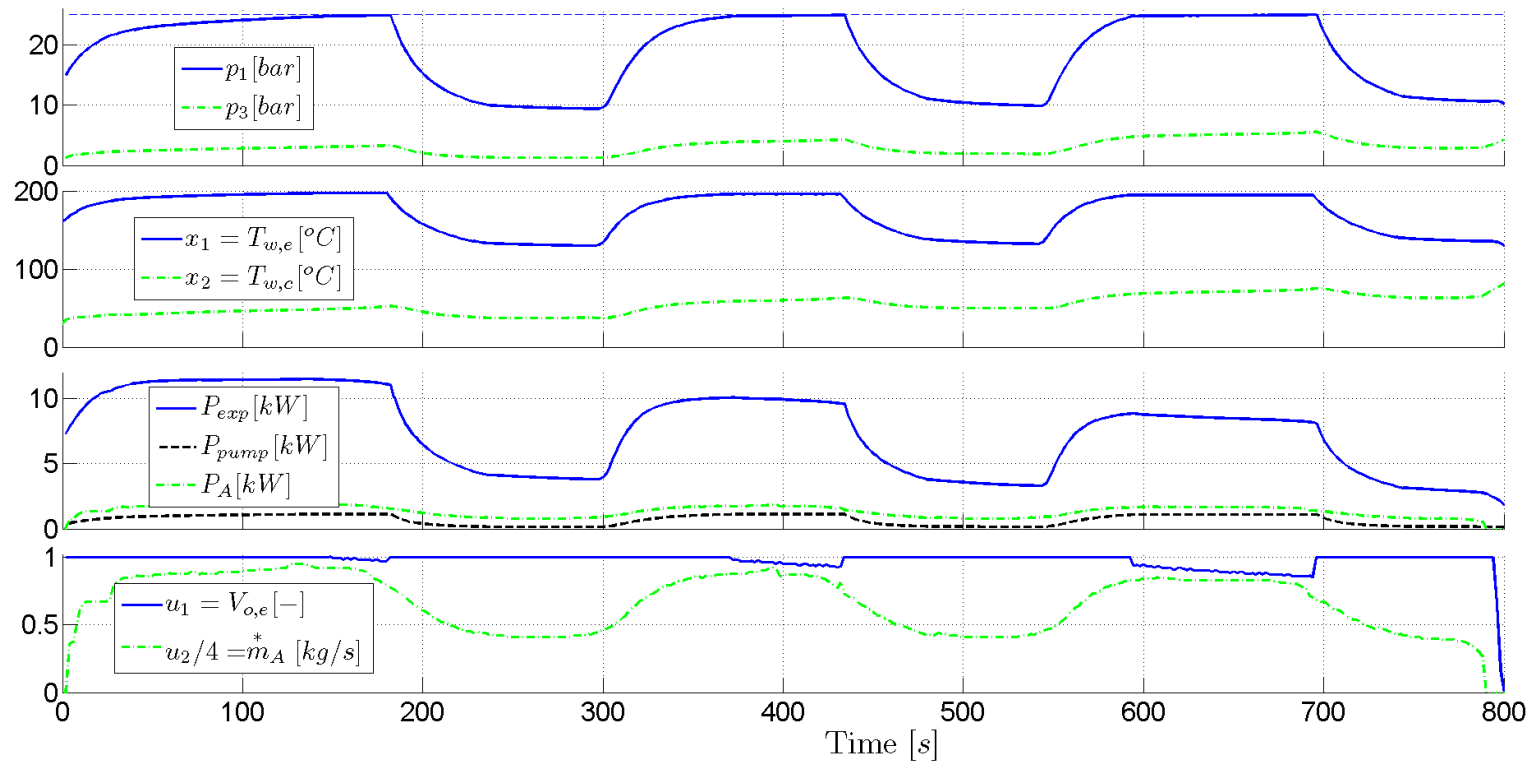

Fig. 12. Adaptive-grid DP solution: time evolution of the ORC system characteristics

set algorithm in different contexts than those presented in the paper.

Nonetheless, the accuracy obtained on the ORC study case with a relatively short computational time opens up new opportunities. For instance, it becomes possible to carry out a thorough parametric study of the ORC system, in order to optimize its design by taking into account the transient behavior.

\section{REFERENCES}

Back, M., Terwen, S., and Krebs, V. (2004). Predictive powertrain control for hybrid electric vehicles. In First IFAC Symposium on Advances in Automotive Control.

Bao, J. and Zhao, L. (2013). A review of working fluid and expander selections for organic rankine cycle. Renewable and Sustainable Energy Reviews, 24(0), 325 - 342.

Bellman, R. (1957). Dynamic programming. Princeton Univ. Press.

Cheng, T., He, X., and Asada, H. (2004). Nonlinear observer design for two-phase flow heat exchangers of air conditioning systems. In American Control Conference, 2004. Proceedings of the 2004, 1534-1539.

Elbert, P., Ebbesen, S., and Guzzella, L. (2013). Implementation of dynamic programming for $\mathrm{n}$-dimensional optimal control problems with final state constraints. IEEE Trans. Control Syst. Technol., 21(3), 924-931.

Endo, T., Kawajiri, S., Kojima, Y., Takahashi, K., Baba, T., Ibaraki, S., Takahashi, T., and Shinohara, M. (2007). Study on maximizing exergy in automotive engines. In SAE World Congress. SAE International.

Guzzella, L. and Sciarretta, A. (2007). Vehicle propulsion systems. Springer.

Manente, G., Toffolo, A., Lazzaretto, A., and Paci, M. (2013). An organic rankine cycle off-design model for the search of the optimal control strategy. Energy, 58(1), $97-106$.

McKinley, T.L. and Alleyne, A.G. (2008). An advanced nonlinear switched heat exchanger model for vapor compression cycles using the moving-boundary method.
International Journal of Refrigeration, 31(7), 1253 1264.

Peralez, J., Tona, P., Sciarretta, A., Dufour, P., and Nadri, M. (2012). Towards model-based control of a steam rankine process for engine waste heat recovery. In Vehicle Power and Propulsion Conference (VPPC), 289-294. IEEE.

Peralez, J., Tona, P., Lepreux, O., Sciarretta, A., Voise, L., Dufour, P., and Nadri, M. (2013). Improving the control performance of an organic rankine cycle system for waste heat recovery from a heavy-duty diesel engine using a model-based approach. In 2013 IEEE Conference on Decision and Control (CDC). Accepted.

Petit, N. and Sciarretta, A. (2011). Optimal drive of electric vehicles using an inversion-based trajectory generation approach. In 18th IFAC World Congress, 1451914526. Milano, Italy.

Powell, W.B. (2007). Approximate Dynamic Programming: Solving the curses of dimensionality, volume 703. John Wiley \& Sons.

Serrao, L., Onori, S., and Rizzoni, G. (2011). A comparative analysis of energy management strategies for hybrid electric vehicles. Journal of Dynamic Systems, Measurement, and Control, 133(3), 031012-031012-9.

Sprouse, C. and Depcik, C. (2013). Review of organic rankine cycles for internal combustion engine exhaust waste heat recovery. Applied thermal engineering, 51(12), 711-722.

Stanton, D. (2013). Systematic development of highly efficient and clean engines to meet future commercial vehicle greenhouse gas regulations. SAE Int. J. Engines, 6(3), 1395-1480.

Sundström, O., Ambühl, D., and Guzzella, L. (2010). On implementation of dynamic programming for optimal control problems with final state constraints. Oil \& Gas Science and Technology-Rev. IFP, 65(1), 91-102.

Xie, H. and Yang, C. (2013). Dynamic behavior of rankine cycle system for waste heat recovery of heavy duty diesel engines under driving cycle. Applied Energy, 112(0), 130 $-141$. 